\title{
Extended Spectrum $\beta$-Lactamase (ESBL) Producing Multidrug Resistant Gram-Negative Bacteria from Various Clinical Specimens of Patients Visiting a Tertiary Care Hospital
}

\author{
Albert Ghimire1, Bipesh Acharya ${ }^{2}$, Reshma Tuladhar ${ }^{*}$ \\ ${ }^{1}$ Central Department of Microbiology, Tribhuvan University, Kirtipur, Kathmandu, Nepal \\ ${ }^{2}$ Head of Pathology Laboratory, Shahid Gangalal National Heart Centre, Bansbari, Kathmandu, Nepal
}

*Corresponding author: Reshma Tuladhar, Central Department of Microbiology, Tribhuvan University, Kirtipur, Nepal; Email: reshma.tuladhar@microbiotu.edu.np

\begin{abstract}
Objectives: The purpose of this study was to assess multidrug resistance and Extended Spectrum $\beta$-Lactamase (ESBL) production in Gram negative bacterial pathogens.

Methods: The study included clinical specimens sent for routine culture and antibiotic susceptibility testing. A total of 469 different clinical specimens were processed according to the standard methodology. The isolates were identified by standard microbiological procedures and subjected to antimicrobial susceptibility testing by modified Kirby-Bauer disk diffusion method. Production of ESBL was determined by combined disk method.

Results: Of the total sample processed, 80 (17.0\%) Gram negative bacteria were isolated and 82.5\% of them were multidrug resistant (MDR). From the total MDR isolates, $47 \%$ were ESBL positive. The higher rate of growth among Intensive Care Units (ICUs) patients was found statistically significant. Higher prevalence of MDR isolates was observed in blood and pus specimens. The majority of the ESBL producers were Escherichia coli (38.7\%). Higher rate of ESBL producers was detected from blood (55.6\%). Polymyxin B, imipenem and amikacin were the most effective antibiotics against Acinetobacter spp. and Pseudomonas aeruginosa whereas imipenem, amikacin, meropenem were the most effective antibiotics against Enterobacteriaceae.
\end{abstract}

Conclusion: Higher prevalence of ESBL producing MDR Gram negative pathogens in hospitalized patients indicates these bacteria are important health care associated pathogens and requires proper infection control measures that check the transfer of MDR and $\beta$-lactamase producing bacterial pathogens among the hospitalized patients.

Key words: Gram negative bacteria, Antibiotic susceptibility testing, MDR, ESBL, Combined disk assay

\section{INTRODUCTION}

Multidrug resistant bacterial infections are spreading worldwide where Extended Spectrum $\beta$-Lactamases (ESBLs) are the major MDR (multidrug resistant) related bacterial enzymes in addition to metallo $\beta$-lactamases, carbapenemases and AmpC $\beta$-lactamases (Chakraborty et al. 2011). The increasing ability to make altered receptors for antimicrobial agents, enzymes to destroy antibiotics and resistant metabolic pathways have significantly increased drug resistances in Gram negative pathogens (Okonko et al. 2009).

Antimicrobial resistance (AMR) is defined as resistance of a microorganism to an antimicrobial medicine to which it was originally sensitive. AMR is a natural phenomenon, which is amplified by continuous and unnecessary exposure to antimicrobials (WHO 2014). According to European Center for Disease Prevention and Control (ECDC), for the Gram negative bacteria such as Acinetobacter baumanii, Pseudomonas aeruginosa, Klebsiella pneumoniae and Escherichia coli, multidrug 
resistance is defined as non-susceptible to at least one agent in at least three different antimicrobial categories. The antimicrobial categories are exclusive for the different organisms (Magiorakos et al. 2012).

ESBLs represent a major group of $\beta$-lactamases which have the ability to hydrolyze and cause resistant to various type of newer $\beta$-lactam antibiotics including the extended-spectrum (or third-generation) cephalosporins and monobactams (aztreonam) but not the cephamycins (cefoxitin and cefotetan) and carbapenems. They are also inhibited by clavulanate, sulbactam and tazobactam alone or in combination with $\beta$-lactams called $\beta$-lactam/ $\beta$-lactamase inhibitors (Vinodhini et al. 2014). In addition to E. coli and Klebsiellaspp, the production of ESBL has become more common in enteric bacilli e. g. Enterobacter aerogenes, E. cloacae, Serratia marcescens, Morganella morganii, Providentia spp., Citrobacter freundii and C. koserias well as in non-enteric bacilli like $P$. aeruginosa. The ESBLs have also been reported in Acinetobacter spp., Burkholderia cepacia and Alcaligenes fecalis (Stürenburg and Mack 2003; Al-Jasser 2006).

As developed by CLSI, the phenotypic method of ESBL detection involves two steps. The first is a screening test with an indicator cephalosporin which looks for resistance or diminished susceptibility, thus identifying isolates likely to be harboring ESBLs. The second one tests forsynergy between oxyimino cephalosporin and clavulanate, distinguishing isolates with ESBLs from those that are resistant for other reasons (Paterson and Bonomo 2005).

The emergence of drug resistant organisms in both hospitals and community is a major concern. Surveillance studies have provided important information about changes in the spectrum of microbial pathogens and trends in the antimicrobial resistance patterns in nosocomial and community acquired infections and continued monitoring. of antimicrobial resistance patterns in hospitals is essential to guide effective empirical therapy. As the incidence of antimicrobial resistance rises, the costs associated with consequences also rises and hence can be considered an economic burden to society of developing country like Nepal. Antibiotic susceptibility profile and reporting of drug resistant strain especially ESBL producing strains would enlighten the appropriate antibiotic therapy and would help in awareness towards misuse and overuse of antibiotics (Paterson and Bonomo 2005). Thus this study was performed to screen and confirm ESBL producing organism.

\section{MATERIALS AND METHODS}

A prospective study was carried out in Shahid Gangalal National Heart Centre, Bansbari, Kathmandu at the Department of Pathology from 19 November 2014 to 18 May 2015. All the clinical specimens received in microbiology laboratory for routine culture and antibiotic susceptibility testing from all the units of hospital was included in this study.

Culture of different clinical specimens was performed using standard microbiological procedures (Forbes et al. 2007). Isolated colonies from the pure culture were identified by performing Gram staining and the standard conventional biochemical tests. Susceptibility tests of the different clinical isolates towards various antibiotics were performed by modified Kirby-Bauer disk diffusion method for the commonly isolated pathogens using Mueller Hinton Agar (MHA). The isolates resistant to at least one antibiotic in at least three different antimicrobial categories were considered as MDR. MDR isolates in pure culture were preserved in $20 \%$ glycerol containing trypticsoy broth and kept at $-4^{\circ} \mathrm{C}$ until subsequent tests for the presence of ESBL was performed.

The MDR isolates were screened for possible ESBL production using ceftazidime $(30 \mu \mathrm{g})$, cefotaxime $(30 \mu \mathrm{g})$ (CLSI 2014). The screen positive isolates, i.e. showing ceftazidime $<22 \mathrm{~mm}$, cefotaxime $<27 \mathrm{~mm}$ zone of inhibition, were subjected to Combined Disk (CD) test using cefotaxime (30 $\mathrm{gg})$ andcefotaxime $(30 \mu \mathrm{g})$ plus clavulanate $(10 \mu \mathrm{g})$ for confirmation of ESBL production. An increase in zone of diameter of $\geq 5 \mathrm{~mm}$ in the presence of clavulanate was concluded as confirmed ESBL producer.

The data obtained were analyzed using Statistical Package for Social Sciences (IBM SPSS) software (Version 21.0). Chi-square $\left(X^{2}\right)$ test was performed to test the significance of distribution of Gram negative bacteria in OPD, wards and ICU. The $p$ value less than 0.05 was considered to be significant.

\section{RESULTS}

A total of 469 clinical specimens, of which 70 (14.9\%) specimens from OPD, 186 (39.7\%) specimens from various wards and 213 (45.4\%) specimens from ICUs (intensive care units) were included in this study. 
Among the 469 clinical specimens analyzed, 159 (33.9\%) were urine, 121 (25.8\%) were blood, 59 (12.6\%) were Endotracheal tube (ET) tip and secretion, 55 (11.7\%) were sputum, 33 (7\%) were body fluids, 14 (3\%) were Central venous pressure (CVP) tip, 12 (2.6\%) were tissue, $11(2.3 \%)$ were pus and $5(1.1 \%)$ were suction tip specimens (Table 1).

Table 1: Distribution of total clinical specimens under investigation

\begin{tabular}{lcc}
\hline Types of specimens & No. of specimens received & Percentage \\
\hline Urine & 159 & 33.9 \\
Blood & 121 & 25.8 \\
ET tip and secretion & 59 & 12.6 \\
Sputum & 55 & 11.7 \\
Body fluids & 33 & 7 \\
CVP tip & 14 & 3 \\
Tissue & 12 & 2.6 \\
Pus & 11 & 2.3 \\
Suction tip & 5 & 1.1 \\
Total & 469 & 100 \\
\hline
\end{tabular}

A total of $80(17.0 \%)$ isolates of eight different type of Gram negative bacteria were isolated from a total of 469 clinical specimens processed, of which 52 isolates belong to family Enterobacteriaceae, 15 isolates were Acinetobacter spp. and 13 isolates were P. aeruginosa. Among the total isolates of Gram negative bacteria $E$. coli was the predominant isolate with $35 \%$ followed by K. pneumoniae (22.5\%), Acinetobacter spp. (18.8\%), P. aeruginosa (16.3\%), Klebsiella oxytoca (2.5\%), Citrobacter freundii $(2.5 \%)$ and $1.2 \%$ each of Proteus mirabilis and Morganella morganii (Figure1).

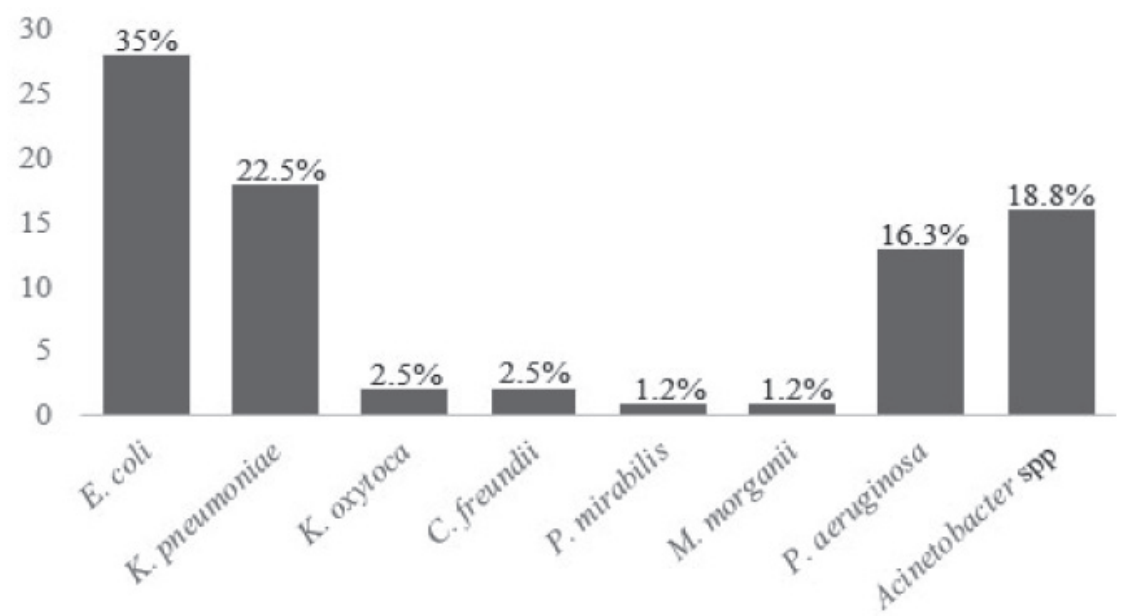

Figure 1: Frequency of different bacteria isolated

Of the total 80 Gram negative bacterial isolates, 52 (65\%) from ICUs, 14 (17.5\%) from wards and 14 (17.5\%) from OPD were isolated. From the $\chi^{2}$ test, it was found that the Gram negative bacteria isolated from OPD, wards and ICUs was statistically significant $(\mathrm{p}<0.05)$ (Table 2$)$.

All the isolated strains of Gram negative bacteria from different clinical specimens were tested with specific antibiotics by using modified Kirby-Bauer disk diffusion method. According to CLSI 2014, 3 different sets of antibiotics were used to determine antibiotic susceptibility pattern of $P$. aeruginosa, Acinetobacter spp. and member of Enterobacteriaceae family. Amikacin and polymyxin B were the most effective drugs with $100 \%$ sensitivity against all the isolates of $P$. aeruginosa followed by imipenem $(84.6 \%)$, piperacillin-tazobactam $(76.9 \%)$ and piperacillin $(61.5 \%)$ while ceftazidime, 
Table 2: Distribution of Gram negative bacteriain OPD, wards and ICUs

\begin{tabular}{lccccc}
\hline \multirow{2}{*}{ Organisms } & \multicolumn{3}{c}{ No. of isolates (\%) } & \multirow{2}{*}{ Total (\%) } & P-value \\
\cline { 2 - 4 } & OPD & Wards & ICUs & & \\
\hline E. coli & $11(39.3)$ & $7(25)$ & $10(35.7)$ & 28 & \\
K. pneumonia & $2(11.1)$ & $4(22.2)$ & $12(66.7)$ & 18 & \\
K. oxytoca & $0(0)$ & $0(0)$ & $2(100)$ & 2 & \\
C. freundii & $0(0)$ & $1(50)$ & $1(50)$ & 2 & $<0.05$ \\
P. mirabilis & $0(0)$ & $1(100)$ & $0(0)$ & 1 & \\
M. morgani & $1(100)$ & $0(0)$ & $0(0)$ & 1 & \\
P. aeruginosa & $0(0)$ & $1(7.7)$ & $12(92.3)$ & 13 & \\
Acinetobacter spp. & $0(0)$ & $0(0)$ & $15(100)$ & 15 & \\
\hline Total & $14(17.5)$ & $\mathbf{1 4 ( 1 7 . 5 )}$ & $\mathbf{5 3 ( 6 5 )}$ & 80 & \\
\hline
\end{tabular}

cefotaxime, aztreonam, meropenem, gentamicin and ciprofloxacin were least effective antibiotics. Similarly, Acinetobacter spp. showed $100 \%$ sensitivity towards polymyxin B while among other antibiotics imipenem andamikacin were found effective against them and $100 \%$ isolates of Acinetobacter spp. were resistant to tetracycline. Imipenem with $100 \%$ susceptibility followed by amikacin, meropenem, gentamicin, chloramphenicol and piperacillin-tazobactam were most effective antibiotics while ampicillin was least effective antibiotic against members of Enterobacteriaceae. Nitrofurantoin was found $80 \%$ effective towards urinary isolates of Enterobacteriaceae.

Of the total 80 isolates of Gram negative bacteria, $66(82.5 \%)$ isolates were MDR. Though E. coli was predominant bacteria among total MDR isolates, the highest percentage of MDR strains among each bacterial isolates were $K$. oxytoca, P. mirabilis and C. freundii with $100 \%$ multidrug resistance each followed by K. pneumoniae (94.4\%) and Acinetobacter spp. (93.3\%). A single isolate of $M$. morganii was non MDR. Of the total 66 MDR isolates, 58 (87.9\%) MDR isolates were suspected of being producer of ESBL. Of the total 58 screen positive isolates for ESBL production, 31 $(53.4 \%)$ isolates were found to be ESBL producer. The prevalence of ESBL producer among total isolates was $38.8 \%(31 / 80)$ whereas the prevalence of ESBL producers among MDR isolates was $47 \%$. Both the isolates of $K$. oxytoca were ESBL producer while a single isolate of MDR P. mirabilis was non ESBL producer. Among the total ESBL positive isolates E. coli was the most predominant isolate with $38.7 \%$ (12/31) followed by $K$. pneumoniae $25.8 \%$ (8/31), P. aeruginosa $16.1 \%$ (5/31), Acinetobacter spp. 9.7\% (3/31), K. oxytoca $6.5 \%$ (2/31) and C. freundii 3.2\% (1/31) (Table 3).

High prevalence of MDR isolates was observed in blood and pus with $100 \%$ multidrug resistance which were followed by ET tip and secretion (95.7\%), sputum

Table 3: Multidrug resistance and ESBL production profile of Gram negative isolates

\begin{tabular}{lcccccc}
\hline Organisms isolated & Total & No. of MDR & No. of suspected & \multicolumn{4}{c}{ Confirmed cases of ESBL } \\
\cline { 6 - 7 } & isolates & strain (\%) & ESBL producer & No. & $\begin{array}{c}\text { \% among } \\
\text { total isolates }\end{array}$ & $\begin{array}{c}\text { \% among MDR } \\
\text { strains }\end{array}$ \\
\hline E. coli & 28 & $21(75)$ & 15 & 12 & 42.9 & 57.1 \\
K. pneumonia & 18 & $17(94.4)$ & 15 & 8 & 44.4 & 47.1 \\
K. oxytoca & 2 & $2(100)$ & 2 & 2 & 100 & 100 \\
C. freundii & 2 & $2(100)$ & 2 & 1 & 50 & 50 \\
P. mirabilis & 1 & $1(100)$ & 1 & 0 & 0 & 0 \\
M. morganii & 1 & $0(0)$ & 0 & 0 & 0 & 0 \\
P. aeruginosa & 13 & $9(69.2)$ & 9 & 5 & 38.5 & 55.6 \\
Acinetobacter spp. & 15 & $14(93.3)$ & 14 & 3 & 20 & 21.4 \\
\hline Total & $\mathbf{8 0}(100)$ & $\mathbf{6 6}(82.5)$ & 58 & 31 & 38.8 & 47 \\
\hline
\end{tabular}


(76.9\%), urine $(74.2 \%)$ and suction tip (50\%). A single isolate from CVP tip was non MDR. High prevalence of ESBL producer was observed in blood (55.6\%) followed by suction tip $(50 \%)$, sputum $(46.2 \%)$, ET tip and secretion $(39.1 \%)$ and urine (32.3\%). No ESBL producer was detected in CVP tip and pus specimens (Table 4).

Table 4: MDR Gram negative isolates in different clinical specimens and their ESBL production profile.

\begin{tabular}{lccc}
\hline Specimens & Total isolates & No. of MDR strains (\%) & No. of ESBL positive isolates (\%) \\
\hline Urine & 31 & $23(74.2)$ & $10(32.3)$ \\
Blood & 9 & $9(100)$ & $5(55.6)$ \\
ET tip and secretion & 23 & $22(95.7)$ & $9(39.1)$ \\
Sputum & 13 & $10(76.9)$ & $6(46.2)$ \\
CVP tip & 1 & $0(0)$ & $0(0)$ \\
Pus & 1 & $1(100)$ & $0(0)$ \\
Suction tip & 2 & $1(50)$ & $1(50)$ \\
\hline Total & 80 & $66(82.5)$ & $31(38.8)$ \\
\hline
\end{tabular}

\section{DISCUSSION}

The emergence of Gram negative bacterial species with acquired resistance to various broad spectrum $\beta$-lactams and other classes of antimicrobials is becoming a worldwide clinical problem. Furthermore, bacteria responsible for causing nosocomial infections are MDR strains, complicating the treatment process (Guthrie 2001).

The prevalence of Gram negative bacteria in various clinical specimens was found to be $17.0 \%$ while that of multidrug resistance was $82.5 \%$. Similar study conducted in National Kidney Center by Panta (2013) showed $19.92 \%$ growth and $85.83 \%$ of them were MDR. However in a study by Upadhyaya (2015) high growth positivity of $27.45 \%$ was observed but the multidrug resistance among the isolates was $77.55 \%$. Another study conducted by Poudyal (2010) showed 19.61\% growth and $61.27 \%$ MDR among isolates.

Of the total 80 Gram negative isolates, E. coli (35\%) was the predominant pathogen followed by K. pneumoniae (22.5\%). Similar results was observed by Bomjan (2005), Maharjan (2010) and Upadhyaya (2015). In comparison of $8.1 \%$ of Acinetobacter spp. isolated by Upadhyaya (2015), 18.8\% of Acinetobacter spp. was isolated in our study. But higher prevalence of $P$. aeruginosa (29.5\%) was found in Upadhyaya (2015) in comparison to our study $(16.3 \%)$.

The highest percentage of MDR strains among each bacterial isolates were K. oxytoca, P. mirabilis and C. freundii with $100 \%$ multidrug resistance each followed by K. pnuemoniae (94.4\%), Acinetobacter spp. (93.3\%), E. coli $(75 \%)$ and $P$. aeruginosa $(69.2 \%)$. These results resembled with the outcomes of previous studies by Poudyal (2010), Mishra et al. (2012), Thakur (2012), Koirala (2014) and Upadhyaya (2015).

High drug resistance in Enterobacteriaceae is attributed to mutations in chromosomal genes, ability to share genetic material and mobile resistant genes. The mobile genetic elements are responsible for capturing resistant genes from the chromosomes of a variety of bacterial species and moving them between DNA molecules horizontally and vertically (Partridge 2015).

The high level of drug resistance seen among $E$. coli is mediated by $\beta$-lactamases, which hydrolyze the $\beta$-lactam ring inactivating the antibiotic. The classical TEM-1, TEM-2, and SHV-1 enzymes are the predominant plasmid-mediated $\beta$-lactamases of Gram negative rods (Livermore 1995). Higher level of drug resistance seen among K. pneumoniae and Acinetobacter spp. is mediated by the production of different kind of $\beta$-lactamases primarily ESBL, AmpC and MBLs. The fact that the carriage of resistance trait for quinolones and aminoglycoside in the plasmid along with the gene for $\beta$-lactamases have had a great impact on the drug resistance character shown by these pathogenic bacteria (Thomson and Moland 2000; Picao et al. 2003; Walsh et al. 2005 Lee et al. 2008). The multidrug efflux systems, inactivation and modification of antibiotics and changes in target sites for antibiotics are the major mechanisms for antibiotic resistance in $P$. aeruginosa (Lambert 2002).

The prevalence of ESBL producers among the total isolates in our study was $38.8 \%(31 / 80)$ whereas the prevalence of ESBL producers among total MDR 
isolates was 47\% (31/67). In similar studies Batchoun et al. (2009), Balan (2013), Thenmozhi and Sureshkumar (2013) and Vinodhini et al. (2014) reported 22.9\%, 23\%, $17.7 \%$ and $54.31 \%$ ESBL producer respectively from total Gram negative bacterial isolates. Among the total 31 ESBL positive isolates, majority of them were E. coli with $38.7 \%$ followed by K. pneumoniae $25.8 \%$, P. aeruginosa $16.1 \%$, Acinetobacter spp. 9.7\%, K. oxytoca $6.5 \%$ and C. freundii $3.2 \%$.

In this study higher prevalence of MDR isolates was observed in blood and pus with 100\% multidrug resistance each. Similar to this study $100 \%$ blood isolates were MDR (Upadhyaya 2015) however only 33.6\% (Dantas et al. 2014) and 18.6\% (Tsai et al. 2014) bacteremia was caused by MDR Gram negative bacteria. In comparison to this study (76.9\%) only $60 \%$ and $37 \%$ MDR isolates from sputum were reported by Poudyal (2010). Panta (2013) and Upadhyaya (2015) reported $88.9 \%$ and $83.3 \%$ MDR isolates respectively of the total urinary isolates while that of only $64.6 \%$ and $38.6 \%$ of the total urinary isolates were reported by Poudyal (2010) and Poudel (2013) respectively.

Higher prevalence of ESBL producer was observed in blood (55.6\%) followed by suction tip (50\%), sputum (46.2\%), ET tip and secretion (39.1\%) and urine (32.3\%). Jagdeesh et al. (2014) reported among screen positive isolates for ESBL, $45.1 \%, 46.7 \%$ and $29.4 \%$ ESBL producers from urine, exudates/pus and sputum respectively while 100\% ESBL producers were detected in stool. However, among total ESBL positive isolates Sharma et al. (2013) reported high prevalence of ESBL producer from respiratory tract specimens (63.83\%) followed by stool $(59.29 \%)$, urine $(57.2 \%)$, body fluid $(52.17 \%)$, pus $(48.03 \%)$ and blood $(31.07 \%)$. Similarly $75 \%, 66.7 \%$ and $25 \%$ of the isolates from urine, exudates and blood were ESBL positive (Umadevi et al. 2011).

The positive ESBL screening result may be more often due to AmpC $\beta$-lactamases than ESBL. It is difficult to detect ESBLs in those isolates that typically have inducible AmpC chromosomal enzyme which may be induced by clavulanate and attack the indicator cephalosporin, thus masking any synergy arising from ESBL production (Livermore and Brown 2001).

In this study the prevalence of MDR Gram negative isolates among the total isolates was high (82.5\%). Among the total MDR isolates $47 \%$ were ESBL positive. Although most of the isolates in the present study were susceptible to carbapenem antibiotics, the resistance shown by some isolates towards this group of antibiotic indicates presence of carbapenemase $\beta$-lactamases in them which requires further characterization.

In addition to this study, observation of higher number of Gram negative bacteria, multidrug resistance and ESBL producing isolates among hospitalized patients in different studies conducted in Nepal indicate MDR Gram negative bacteria are emerging as important health care associated pathogens (Panta et al. 2013; Mishra et al. 2014; Parajuli et al. 2017). Thus it is essential for tertiary care hospitals of Nepal to perform routine detection of ESBLs and other $\beta$-lactamases.

Most of the ESBL producing bacteria show multi drug resistance pattern creating a therapeutic dilemma for the clinicians. It is very important to determine the preferable antibiotics for the treatment. Infection control measures, hygiene guidelines, appropriate antibiotic policies that control the widespread use of advanced cephalosporins are immediately required to prevent and to ameliorate the ever increasing problem of the emergence of MDR ESBL producing Gram negative bacteria (Giamarellou 2005).

\section{CONCLUSION}

Presence of ESBL producing MDR Gram negative pathogens in patients of different department of tertiary care hospital of Nepal indicates these bacteria are important health care associated pathogens which can pose threat to the treatment. Thus, a proper infection control measure is required to check the transfer of MDR and $\beta$-lactamase producing bacterial pathogens among the hospitalized patients.

\section{ACKNOWLEDGEMENTS}

We are most grateful to the managing director of Shahid Gangalal National Heart Centre for giving us the clinical facilities and the necessary support. We would like to thank all the staff of the SGNHC for their support and suggestions.

\section{REFERENCES}

Al-Jasser AM (2006) Extended spectrum $\beta$-lactamases (ESBLs): a global problem. Kuwait Med J 38: 171185.

Balan K (2013) Detection of extended spectrum $\beta$-lactamase among Gram negative clinical isolates from a tertiary care hospital in South India. Int J Res Med Sci 1: 28-30. 
Batchoun RG, Swedan SF and Shurman AM (2009) Extended spectrum $\beta$-lactamases among Gram negative bacterial isolates from clinical specimens in three major hospitals in Northern Jordan. Int J Microbiol 513874.

Bomjan R (2005) Prevalence of multidrug resistant strains with reference to extended spectrum $\beta$-lactamase producing strains among the bacterial pathogens isolated from different clinical samples at Tribhuvan University Teaching Hospital. M. Sc. Dissertation submitted to the Central Department of Microbiology, Tribhuvan University. pp 44-72.

Chakraborty D, Basu S and Das S (2011) Study on some Gram negative multidrug resistant bacteria and their molecular characterization. Asian J Pharm Clin Res 4: 108-112.

Dantas RCC, Gontijo-Filho PP, Btistao DWF, Ferreira ML and Ribas RM (2014) Bacteremia due to resistant Gram negative bacilli: risk factors and impact of resistance on outcome Rev Panam Infectol 16: 206-214.

Forbes BA, Sahm DF and Weissfeld AS (2007) Bailey and Scott's diagnostic Microbiology $12^{\text {th }}$ edition. Mosby Elsevier Publication, USA pp 534-678.

Giamarellou H (2005) Multidrug resistance in Gram negative bacteria that produce extended spectrum $\beta$-lactamases (ESBLs). Clin Microbiol Infect 11: 1-16.

Guthrie R (2001) Community-acquired lower respiratory tract infections: etiology andtreatment. CHEST 120: 2021-2034.

Jagdeesh VS, Mahalakshmi VV, Hajare V, Kumar A and Sreekantha H (2014) Prevalance and antibiogram of extended spectrum $\beta$-lactamase producing E. coli, Klebsiella and Pseudomonas species from clinical isolates in a tertiary care hospital. Res J Pharm Biol Chem Sci 5: 258-274.

Koirala A (2014) Extended spectrum ßlactamse (ESBL) and metallo $\beta$-lactamse (MBL) mediated resistance in clinical isolates of non fermenting Gram negative bacilli (NFGB). M. Sc. Dissertation submitted to the Central Department of Microbiology, Tribhuvan University. pp 32-45.

Lambert PA (2002) Mechanisms of antibiotic resistance in P. aeruginosa. J R Soc Med 95: 22-26.
Lee K, Lim YS, Yong D, Yum JH and Chong Y (2003) Evaluation of the Hodge test and the imipenemEDTA double-disk synergy test for differentiating metallo- $\beta$-lactamase-producing isolates of Pseudomonas spp. and Acinetobacter spp. J Clin Microbiol 41: 4623-4629.

Livermore DM (1995) $\beta$-lactamases in laboratory and clinical resistance. Clin Microbiol Rev 8: 557-584.

Livermore DM and Brown DFJ (2001) Detection of $\beta$-lactamase-mediated resistance. I Antimicrob Chemother 48: 59-64.

Magiorakos AP, Srinivasan A, Carey RB, Carmeli Y, Falagas ME, Giske CG, Harbarth $S$ and Monnet DL (2012) Multi drug resistant, extensively drug resistant and pan drug resistant bacteria: an international expert proposal for interim standard definitions for acquired resistance. Clin Microbiol Infect 18: 268-281.

Maharjan S (2010) Multidrug resistance and extended spectrum $\beta$-lactamases producing strains among clinical isolates of patients from Shree Birendra hospital, Chhauni. M. Sc. Dissertation submitted to the Central Department of Microbiology, Tribhuvan University. pp 51-65.

Mishra SK, Acharya J, Kattel HP, Koirala J, Rijal BP and Pokhrel BM (2012) Metalloß-lactamase producing Gram negative bacterial isolates. J Nepal Health Res Counc 10: 208-213.

Okonko IO, Soleye FA, Amusan TA, Ogun AA, Ogunnusi TA, Ejembi J, Egun OC and Onajobi BI (2009) Incidence of multi-drug resistance (MDR) organisms in Abeokuta, Southwestern Nigeria. Global J Pharmacol 3: 69-80.

Panta A (2013) Extended Spectrum $\beta$-lactamase and Metallo $\beta$-lactamaseproducing multidrug resistant Gram negative bacteria among patients with renal failure. M. Sc. Dissertation submitted to Central Department of Microbiology, Tribhuvan University. pp 27-39.

Parajuli NP, Acharya SP, Mishra SK, Parajuli K, Rijal BP and Pokharel BM (2017) High burden of antimicrobial resistance among Gram negative bacteria causing healthcare associated infections in a critical care unit of Nepal. Antimicrob Resist Infect Control 6: 67. 
Partridge SR (2015) Resistance mechanisms in Enterobacteriaceae. Pathology J RCPA 47: 276-284.

Paterson DL and Bonomo RA (2005) Extendedspectrum $\beta$-lactamases: a clinical update. Clinl Microbiol Rev 18: 657-686.

Paudel S (2013) Status of extended spectrum $\beta$-lactamase producing Enterobacteriaceae among uropathogens. M. Sc. Dissertation submitted to Central Department of Microbiology, Tribhuvan University. pp 27-36.

Picao RC, Andrade SS, Nicoletti AG, Campana EH, Moraes GC, Mendes RE and Gales AC(2008) Metallo- $\beta$-Lactamase detection: Comparative evaluation of double disk synergy versus combined disk tests for IMP-, GIM-, SIM-, SPM-, or VIM- producing isolates. J Clin Microbiol 46: 2028- 2037.

Poudyal S (2010) Prevalence of $\beta$-lactamase producing multidrug resistant bacterial pathogens isolated from different clinical samples at National Public Health Laboratory. M. Sc. Dissertation submitted to Central Department of Microbiology, Tribhuvan University. pp 44-58.

Sharma M, Pathak S and Srivstava P (2013) Prevalence and antibiogram of Extended Spectrum $\beta$-Lactamase (ESBL) producing Gram negative bacilli and further molecular characterization of ESBL producing Escherichia coli and Klebsiella spp. J Clin Diag Res 7: 2173-2177.

Stürenburg E and Mack D (2003) Extended-spectrum $\beta$-lactamases: implications for the clinical microbiology laboratory, therapy and infection control. J Infect 47: 273-295.

Thakur P (2012) Multidrug resistant bacterial isolates at Nobel Medical College Teaching Hospital. M. Sc. Dissertation submitted to the Central Department of Microbiology, Tribhuvan University. pp 36-50.

Thenmozhi S and Sureshkumar BT (2013) Prevalence of Extended spectrum $\beta$-actamase producing
Gram negative bacteria in private hospital, Tiruchengode, Tamilnadu, India. Int J Curr Microbiol App Sci 2: 280-289.

Thomson KS and Smith Moland E (2000) Version 2000: the new $\beta$-lactamases of Gram negative bacteria at the dawn of the new millennium. Microbes Infect 2: 1225-1235.

Tsai MH, Chu SM, Hsu JF, Lien R, Huang HR, Chiang MC, Fu RH, Lee CW and Huang YC (2014) Risk factors and outcomes for multidrug-resistant Gram negative bacteremia in the NICU. Pediatrics 133: e322-e329.

Umadevi S, Kandhakumari G, Joseph NM, Kumar S, Easow JM, Stephen S and Singh UK (2011) Prevalence and antimicrobial susceptibility pattern of ESBL producing Gram negative bacilli. J Clin Diagn Res 5: 236-239.

Upadhyaya U (2015) Detection of $\beta$-lactamase producing Gram negative bacteria in different clinical specimens of patients visiting tertiary level heart center. M. Sc. Dissertation submitted to Central Department of Microbiology, Tribhuvan University. pp 28-36.

Vinodhini R, Moorthy K, Palanivel P, Punitha T, Saranya S, Bhuvaneshwari M and Kanimozhi C (2014) Detection and antimicrobial susceptibility pattern of ESBL producing Gram negative bacteria. Asian J Pharm Clin Res 7: 243-247.

Walsh TR, Toleman MA, Poirel L and Nordmann P (2005) Metallo- $\beta$-lactamases: the quiet before the storm? Clin Microbiol Rev 18: 306-325.

World Health Organization (2014) Antimicrobial resistance: global report on surveillance. World Health Organization. 